\title{
Potential Role of Leptin Signaling in DMBA-induced Mammary Tumors by Non-Responsive C57BL/6J Mice Fed a High-Fat Diet \\ Gillespie $C^{1}$, Quarshie A $^{2}$, Penichet $\mathbf{M}^{3}$ and Gonzalez-Perez RR ${ }^{1 *}$
}

${ }^{1}$ Department of Microbiology, Biochemistry \& Immunology, Morehouse School of Medicine, Atlanta, USA

${ }^{2}$ Biomedical Informatics Program and Master of Science in Clinical Research Program, Clinical Research Center, Morehouse School of Medicine, Atlanta, USA ${ }^{3}$ Omnipathology, Pasadena, USA

\begin{abstract}
Environmental carcinogens, High-Fat Diet (HFD) and elevated levels of leptin correlate to increase breast cancer incidence. To test whether these factors could affect the development of Mammary Tumors (MT) via DMBA (7,12-dimethylbenz[a]anthracene) challenge, we used C57BL/6J mice that are non-responsive to develop MT in absence of hormonal stimulation. C57BL/6J female mice without hormonal stimulation were fed HFD (55\% cal-fat) and low-fat diets (10\% cal-fat) and received DMBA (oral gavage: $1 \mathrm{mg} /$ weekly) for 6 weeks. To test whether leptin signaling is involved in DMBA-MT development, a potent inhibitor, pegylated leptin peptide receptor antagonist (PEGLPrA2; half-life 66 hours), was used for 30 weeks. As expected, irrespective of PEG-LPrA2 treatment, lean mice fed with low-fat diet did not develop MT. However, HFD induced obesity and significantly stimulated earlier onset (within 18 weeks) and marginally increased the incidence of MT $(21 \% ; 3 / 14)$ in DIO-mice (diet-induced-obesity). It appears that leptin signaling may be involved in DMBA-induced mammary carcinogenesis in obese mice because no evidence of MT was found in DIO-mice treated with PEG-LPrA2 ( $\%$ incidence; 0/14; Wilcoxon-Breslow test Chi2, $p=0.03$ ). Interestingly, PEG-LPrA2 treatment did not apparently affect body weight or food intake, but reduced protein levels of several molecules related to breast cancer [Aryl hydrocarbon Receptor (AhR), leptin receptor (OB$\mathrm{R})$, interleukin 1 receptor type I (IL-1R tl), hypoxia-induced factor 1 alpha (HIF-1 $\alpha$ ), Jagged1 (JAG1) and Notch1 activated (NICD1)] within the mammary glands. Our findings reinforce the idea that obesity induced by HFD is an additional risk factor for chemical-induced breast carcinogenesis. The present study reveals some potential mechanisms involving leptin in the effects of HFD and adiposity on mammary chemical-induced carcinogenesis. Overall, present data suggest that the inhibition of leptin signaling might be a new way to prevent breast cancer induced by chemical carcinogens, especially in obese individuals.
\end{abstract}

Keywords: High-fat diet; Leptin; Leptin-peptide receptor antagonist; PEG-LPrA2; DMBA; Obesity; Breast cancer prevention

\section{Introduction}

DMBA (7,12-dimethylbenz[a]anthracene), a Polycyclic Aromatic Hydrocarbon $(\mathrm{PAH})$, is a proven carcinogen widely used for research in breast cancer [1]. PAH can induce several mutations promoting cancers of the breast, skin, pancreas, lung, immune system and others [2,3]. PAH-activated Aryl hydrocarbon Receptor (AhR) forms a complex with the nuclear translocator protein (ARNT) inducing phase-I detoxification enzymes (cytochrome P450: CYP1A1 and CYP1B1). These enzymes transform DMBA into toxic and mutagenic intermediates (i.e., pro-DNA adduct compound, epoxide intermediate), which induce carcinogenesis-promoting DNA mutations [1].

There is evidence that the development of DMBA-induced tumors require other factors that can act in a cell-context manner [4-6]. Breast cancer rates vary widely in different parts of the world. Environmental contaminants can be related to breast cancer incidence. Humans are exposed to thousands of naturally occurring and synthetic chemicals over a lifetime. Environmental Protection Agency (EPA) has suggested that exposure to environmental chemicals has been offered as possible explanation for higher incidence of breast cancer in certain regions. For example, various halogenated hydrocarbons and pesticides have been demonstrated to cause a variety of adverse health effects, including increase of breast cancer risk [7,8]. In addition, High-Fat Diet (HFD) or the Western diet and obesity are also positively correlated to breast cancer incidence. Obesity, characterized by high levels of leptin, is pandemic in the United States and associated with more than 100,000 incidents of cancer each year, particularly cancers of the breast, colon and endometrium. Obese breast cancer patients have increased mortality compared with non-obese $[9,10]$.

Chemical carcinogenic challenge in rats has been the most successful model used to demonstrate the effects of pregnancy $[11,12]$ lactation [13] and obesity in mammary tumorigenesis [14]. Intake of low-energy (caloric restriction) or low-fat diets reduces the growth of DMBA-mammary tumors (MT) in rats [15] suggesting a role of obesity/diet in chemical carcinogenic induced-MT. Reported data support the idea that rats fed a HFD and treated with DMBA have a higher positive response to the develop MT [14] when compared to mice under similar experimental conditions. This was true even in research that used mouse strains with high potential to develop MT induced by DMBA [16]. Therefore, the mouse model is more suitable to examine the conditions that can overcome the refractoriness to develop MT induced by carcinogenics [13]. More common methods to induce MT in resistant mice after treatment with carcinogenics include

*Corresponding author: Gonzalez-Perez Ruben R, Department of Microbiology Biochemistry \& Immunology, Morehouse School of Medicine, Atlanta, GA 30310 , USA, E-mail: rgonzalez@msm.edu

Received October 15, 2012; Accepted November 14, 2012; Published November 22, 2012

Citation: Gillespie C, Quarshie A, Penichet M, Gonzalez-Perez RR (2012) Potential Role of Leptin Signaling in DMBA-induced Mammary Tumors by NonResponsive C57BL/6J Mice Fed a High-Fat Diet. J Carcinogene Mutagene 3:132. doi:10.4172/2157-2518.1000132

Copyright: (c) 2012 Gillespie C, et al. This is an open-access article distributed under the terms of the Creative Commons Attribution License, which permits unrestricted use, distribution, and reproduction in any medium, provided the original author and source are credited. 
Citation: Gillespie C, Quarshie A, Penichet M, Gonzalez-Perez RR (2012) Potential Role of Leptin Signaling in DMBA-induced Mammary Tumors by Non-Responsive C57BL/6J Mice Fed a High-Fat Diet. J Carcinogene Mutagene 3:132. doi:10.4172/2157-2518.1000132

natural hormone stimulation (i.e., pregnancy, pseudopregnancy), and supply of hormones or implants of pituitary isografts [17]. Remarkably, C57BL/6J mice without hormonal stimulation are completely irresponsive to the development of DMBA-MT. Interestingly, caloric restriction reduces $\mathrm{BW}$ and increases life-span of $\mathrm{C} 57 \mathrm{BL} / 6 \mathrm{~J}$ mice treated with DMBA, but without MT [18]. This would suggest that reduction of BW (adiposity) decrease DMBA damage in these mice.

Leptin, a key adipokine released from adipose tissue, serves as a major player in the basic homeostatic functions required to regulate appetite and energy expenditure for controlling body weight [19]. Leptin and its receptor, OB-R, are overexpressed in several cancer types, where they induce tumor growth through several actions (proangiogenic, pro-inflammatory and anti-apoptotic) [20-23]. Moreover, proving leptin's oncogenic actions, the inhibition of leptin signaling with pegylated leptin peptide receptor antagonist (PEG-LPrA2) significantly reduced tumor growth and, levels of pro-angiogenic and inflammatory molecules (i.e., VEGF/VEGFR-2, IL-1 system) in syngeneic and xenograft mouse models of breast cancer [20,22].

We hypothesize that obesity induced via HFD will accelerate DMBA-MT development in C57BL/6J mice without hormonal stimulation. Moreover, we postulate that leptin signaling could play a role in DMBA-MT development in obese mice fed HFD. Our results suggest that HFD intake shorten the onset and marginally increased incidence of DMBA-MT in female C57BL/6J mice. Notably, the inhibition of leptin signaling prevented MT development and reduced the levels of several molecules with potential oncogenic roles in breast cancer.

\section{Materials and Methods}

\section{Reagents and antibodies}

Polyclonal Notch1 (sc-373891), Notch4 (sc-56594), Jagged1 (sc8303), OB-R-NH2 (sc-1834), leptin (sc-843), IL-1R tI (sc-689), NFkBp50 (sc-114) and -p65 (sc-109), Bcl-2 (sc-783), Flk-1 (VEGFR-2) (sc6251), VEGF-A (sc-152) antibodies were obtained from Santa Cruz Biotechnology, Inc. (Santa Cruz, CA, USA). Polyclonal NFkB- p105 (ab7971), Notch2 (ab-8926), Notch3 (ab-23426), DLL-4 (ab-7280) and monoclonal AhR (ab2770) antibodies were from Abcam Inc (Cambridge, MA, USA). Polyclonal Hey2 antibody (Ab5716) and insulin ELISA kit ware from Millipore (Temecula, CA, USA). Polyclonal Survivin antibody (2808S) was from Cell Signaling (Danvers, MA, USA). Polyclonal anti-mouse and anti-rabbit antibodies horseradish peroxidase (HRP) conjugates were from Bio-Rad Lab. (Hercules, CA, USA). Recombinant mouse leptin, monoclonal HIF-1a (MAB153) and IL-1 alpha/IL-1F1 and VEGF Quantikine ELISA Kits were purchased from R\&D Systems Inc. (Minneapolis, MN, USA). Estradiol ELISA kit was from Cayman Chemical Corporation (Ann Arbor, MI, USA). ECL Western blot stripping buffer was from Thermo Scientific (Rockford, IL, USA). 7,12-dimethylbenz[a] anthracene (DMBA), monoclonal Notch1 (N6786) and $\beta$-actin (A5316) antibodies, protease inhibitor, phosphatase inhibitor cocktails 1 and 2 and, other chemicals were purchased from Sigma-Aldrich (St. Louis, MO, USA).

\section{Leptin peptide receptor antagonist (PEG-LPrA2)}

The leptin receptor peptide antagonist 2 (LPrA2) with high binding affinity for the leptin receptor $\left(\mathrm{OB}-\mathrm{R} ; \mathrm{Ki} \approx 0.6 \times 10^{10} \mathrm{M}\right.$ ) was synthesized and purified as described elsewhere[24]. To increase its solubility and half-life the peptide was coupled to polyethylene glycol (pegylated peptide: PEG-LPrA2; MW $\approx 23000$; half-life in mice via i.v.: unconjugated 1 hour versus pegylated 66 hours). In contrast to the unconjugated peptide, its derivative, PEG-LPrA2, is water-soluble [22]

\section{Animals and experimental procedures}

Female C57BL/6J mice seven-week old (Jackson Laboratories, Bar Harbor, ME, USA) were randomly allocated into two groups: lean $(n=20)$ and DIO $(n=30)$. Mice were housed three per cage in the animal facilities of Morehouse School of Medicine (MSM, Atlanta, GA, USA) in rooms maintained at $25^{\circ} \mathrm{C}$ with $10-15$ air exchanges per hour. Artificial light was provided under a 12h/12h light/dark cycle. All housing materials, as well as food and water, were autoclaved prior to use. All experiments were performed according to the protocol approved by MSM-IACUC and NIH guide for the Care and Use of Laboratory Animals. The PDI-1 diet (low-fat diet: $10 \%$ Kcal-fat; 4.1 $\mathrm{cal} / \mathrm{g}$ ), free of phytoestrogens (phyto-free; approximately 10-15 ppm of soy isoflavones; Ziegler Bros.; Gardner, PA, USA) was used to feed the lean control mice for 37 weeks. The PDI-1 (Phytoestrogen Reduced I 22.5-5) rodent diet is specifically formulated for studies where dietary concentrations of naturally occurring phytoestrogens, such as genistein, daidzein or glycitein, can influence experimental results. The formulation of this diet is a modification of the NIH-07 diet where ingredients such as soybean products and alfalfa meal, known to contain these hormones, are not used. Main components of the PDI-1 diet include: Corn, Wheat, Fish Meal, Wheat Middlings, Lactic Casein, Dried Skim Milk, Corn Gluten Meal, Corn Oil, Brewers Dried Yeast, Dry Molasses, Limestone, Salt, Dicalcium Phosphate, Vitamin D3 Supplement, dl-Alpha Tocopheryl Acetate (Vitamin E), Folic Acid, Niacin, Riboflavin, Thiamine, Vitamin B12 Supplement, Pyridoxine, Menadione, Sodium Bisulfite Complex (Vitamin K) Vitamin A Palmitate, Choline, Biotin Calcium, Pantothenate, Cobalt Carbonate, Copper Sulfate, Ferrous Sulfate, Manganese Oxide (for more details on PDI diet components see [25]. The PDI-1 diet was modified by adding $24.7 \mathrm{gm} \%$ corn oil (75.3g of PDI plus $24.7 \mathrm{gm}$ of corn oil; total $55 \%$ Kcal-fat; $5.2 \mathrm{cal} / \mathrm{g}$ ). The high-fat diet was used to feed the mice in order to induce obesity (DIO) for 37 weeks. After one week of acclimatization the mice were fed either the lean or DIO diets at libitum. Lean control and DIO-diets were prepared in pellets. Each cage was provided with a supply of pre-weighted diet. Food intake was determined weekly by weighting the differences of remain diets in each cage divide by number of mice ( $\mathrm{n}=3 \mathrm{mice} / \mathrm{cage}$ ). Caloric intake was calculated by multiplying the food intake by the calories per gm (i.e., lean control diet, $4.1 \mathrm{cal} / \mathrm{gm}$; DIO-diet, $5.2 \mathrm{cal} / \mathrm{gm})$. Obesity was identified in DIO-mice showing body weights (BW) greater than BW of lean control group (L-C) plus two standard deviation (SD) as follows: $\mathrm{BW}_{\mathrm{DIO}}>\mathrm{BW}_{\mathrm{L}-\mathrm{C}}+2 \mathrm{SD}$.

\section{PEG-LPrA2 treatment}

Half-life of PEG-LPrA2 is approximately 66 hour, therefore, to establish efficacy the pegylated antagonist was injected into lean and DIO-mice once ('I") or two-times ("II") a week via tail vein $(50 \mu \mathrm{l} / 0.1 \mathrm{mM})$. Mice were randomly allocated into three subgroups each: Lean: control L-C $(\mathrm{n}=6)$ and, treated L-I $(\mathrm{n}=7)$ and L-II $(\mathrm{n}=7)$ and, DIO: control DIO-C $(\mathrm{n}=14)$ and, treated DIO-I $(\mathrm{n}=7)$ and DIO-II $(n=7)$. PEG-LPrA2 treatment was initiated when obesity was detected after 7 weeks fed the DIO diet. PEG-LPrA2 treatment was applied to lean and DIO-mice for 30 weeks. L-C and DIO-C mice received two injections per week of saline (vehicle). Mice fed HFD that did not develop obesity were considered obesity-resistant $(n=2 ; \approx 7 \%)$ and excluded from the studies.

\section{DMBA challenge}

Two weeks after initiated PEG-LPrA2 treatment all mice received 
DMBA ( $1 \mathrm{mg} / 0.2 \mathrm{ml}$ sesame oil; Sigma-Aldrich) by gastric gavage/ weekly for six weeks (total DMBA dose: $6 \mathrm{mg}$ ) [16,26].

\section{Tumor detection}

Mammary glands were palpated weekly for MT detection. Tumor onset, number (multiplicity) and growth were recorded when possible (caliper; $\pi / 6 \mathrm{x}$ width ${ }^{2} \times$ length).

\section{Blood collection and euthanasia}

Blood samples from $8 \mathrm{~h}$-fasted mice were collected in tubes containing EDTA-citrate anticoagulant (Sigma) by cardiac or retroorbital sinus punctures under anesthesia before euthanasia. Mice were euthanized at the end of the experimental period ( 37 weeks of feeding low-fat and HFD, which corresponds to 30 weeks of PEGLPrA2 treatment and 28 weeks after the initiation of DMBA challenge). MTs were dissected and their weight/volume and multiplicity were determined after euthanasia. Mammary gland tissues without apparent MT development were also taken from mice.

\section{Pathology}

AP/CP and Cytopathology board certified pathologist (MP) evaluated the Hematoxilin and Eosin stained preparations of tumors in a blinded protocol.

\section{Immunohistochemistry (IHC)}

IHC was carried-out in paraffin embedded sections from mammary glands and MT $(5 \mu \mathrm{m})$. Briefly, unmasking of tissue antigens was performed by heat treatment in sodium citrate buffer $(\mathrm{pH} 6,10 \mathrm{mM})$ at $95^{\circ} \mathrm{C}$ for 15 minutes and/or partial digestion at $37^{\circ} \mathrm{C}$ for 10 minutes with protease (Sigma Inc.). After quenching endogenous peroxidase activity with $\mathrm{H}_{2} \mathrm{O}_{2}$ (3\% water solution) and blocking (2.5\% horse or rabbit normal serum), tissue sections were incubated for 1 hour at room temperature with the following primary antibodies diluted in PBS- $0.1 \%$ BSA: anti AhR (1:100); OB-R (1:250); leptin (1:50); Flk-1 (VEGFR-2; 1:50); IL-1R tI (1:50); Notch1 (1:50); Notch2 (1:200); Notch3 (1:250); Notch4 (1:50); Jagged1 (JAG1; 1:50); Survivin (1:400); and Bcl-2 (1:50). The tissues were incubated with a streptavidin-biotin peroxidase system according to the manufacturer's directions (Vectastain, ABC-AP kit; Vector, Burlingame, CA, USA), counterstained with hematoxylin (Dako Corp., Carpinteria, CA, USA), and mounted with VectaMount (Vector). Negative controls were also included in which the primary antibody was omitted or substituted with non-specific IgG. Positive cells were recorded and counted in randomly chosen fields. Staining intensity was assigned as weak, moderate, or strong.

\section{Western Blot (WB)}

Tissue lysates from MT and mammary glands from PEG-LPrA2 treated and untreated mice were prepared. Thirty $\mu \mathrm{g}$ of protein lysates were used for WB analysis of several leptin-targeted molecules: Notch/ NICD; Bcl-2; IL-1R tI and AhR as previously described [20]. $\beta$-actin serves as the experimental loading control. For quantitative evaluation of antigen expression, the blots were scanned and analyzed by the NIH Image program [22]. Protein concentrations from lysated tissues were determined by the Bradford method (Bio-Rad).

\section{Immunoprecipitation (IP)}

Fifty micrograms of proteins from tissue lysates were immunoprecipitated with specific antibodies and protein G-agarose beads for IP/WB analysis of VEGFR-2, OB-R, HIF-1 $\alpha$, and Survivin as described elsewhere [22].

\section{Plasma and tissue determinations}

ELISA (R\&D System Inc) determined the levels of IL-1 $\beta$, IL-1 $\alpha$, VEGF and leptin in plasma and tissues lysates. Plasma levels of insulin (Millipore), adiponectin (R\&D Systems Inc) and estradiol (E2; Cayman Chem. Co.) were also determined by ELISA. Glucose levels were determined by glucometer (Accu-Chek Active, Roche Diagnostic, IN, USA).

\section{Statistical Analysis}

All continuous data were summarized using means \pm standard deviation. An ANOVA model was implemented to analyze changes of body weight, food intake and caloric intake between the six subgroups (L-C, L-I, L-II, DIO-C, DIO-I and DIO-II). The effects of HFD and PEGLPrA2 on MT incidence of DIO-mice were analyzed by the Pearson $\mathrm{Chi} 2$ and likelihood-ratio Chi-squared tests. The tumor-free times were plotted using a Kaplan-Meier curve [27] and the median times were compared using the Wilcoxon-Breslow test. Statistical significance was set at $\mathrm{p}<0.05$. Within the lean group, an ANOVA model was also fitted to analyze plasma and mammary tissue parameters between treated and non-treated (control) groups. Within the obese (DIO) group, an ANOVA model was fitted to analyze the relative expression of antigens in mammary tissues and MT, and plasma parameters.

\section{Results}

\section{Impact of diets and treatments on food/caloric intake and body weight}

Figure 1 depicts weekly average of food (FI) (Figure 1A), caloric intake (CI) (Figure 1B) and, body weight (BW) (Figure 1C) in lean untreated control (L-C) and DIO-mice (DIO-C; only receiving saline), and treated mice receiving one (L-I and DIO-I) or two doses (L-II and DIO-II) of PEG-LPrA2. FI was similar between groups independently of diets or treatments (FI: 2-3g/day/mouse) (Figure 1A). However, because diets were not isocaloric, DIO-mice consumed 3-fold more calories during the 37-week experimental period (Figure 1B). Interestingly, DIO-C and DIO-I showed significantly higher CI than DIO-II at week 37 ( $p<0.01$; see Figure 1B). Ninety three percent of mice fed a DIO diet (HFD; 55\% Kcal-fat) were classified as obese $(n=28 / 30)$ after 7 weeks. Obesity was determined using the criterion reported by Dogan et al. [28] as follows: mice fed the HFD with final $\mathrm{BW} \pm 2$ standard deviations of low-fat untreated (control) mice (BW $\left.>\mathrm{BW}_{\mathrm{L}-\mathrm{C}}+2 \mathrm{SD}\right)$. In contrast, only $\approx 7 \%(\mathrm{n}=2 / 30)$ of HFD fed mice were classified as obesity-resistant (OR) using the following criterion: OR $\left(\mathrm{BW}_{\mathrm{OR}} \leq \mathrm{BW}_{\mathrm{L}-\mathrm{C}}+2 \mathrm{SD}\right)$. The OR mice were excluded from the trial. In comparison, the DIO-mouse group gained more weight (increase 199\%) than the compared lean mouse group fed a low-fat diet during the experimental time (37 weeks) (Figure 1C and Table 1). However, due to age progression lean mice significantly increased its average BW (increase 140\%) during the course of this study. However, no significant differences in $\mathrm{BW}$ were found between lean treated or untreated with PEG-LPrA2 at any dose (L-C vs L-I and L-II). Similar results were found in obese (DIO-C vs DIO-I) mice after PEG-LPrA2 treatments (Figure 1C and Table 1). Interestingly, DIO-mice receiving two doses of PEG-LPrA2 (DIO-II) showed significantly lower average BW than DIO-C (BW $\left.{ }_{\text {DIO-II }}<30 \% \mathrm{BW}_{\text {DIO-C; }} \mathrm{p}=0.01\right)$ (Figure $1 \mathrm{C}$ and Table 1).

\section{Effects of HFD and PEG-LPrA2 on onset and incidence of MT}

The effects of HFD and PEG-LPrA2 on DMBA-MT onset and incidence were analyzed using the Wilcoxon-Breslow test in three phases: (1) Comparing MT-free time for all 4 mice sub-groups (lean 

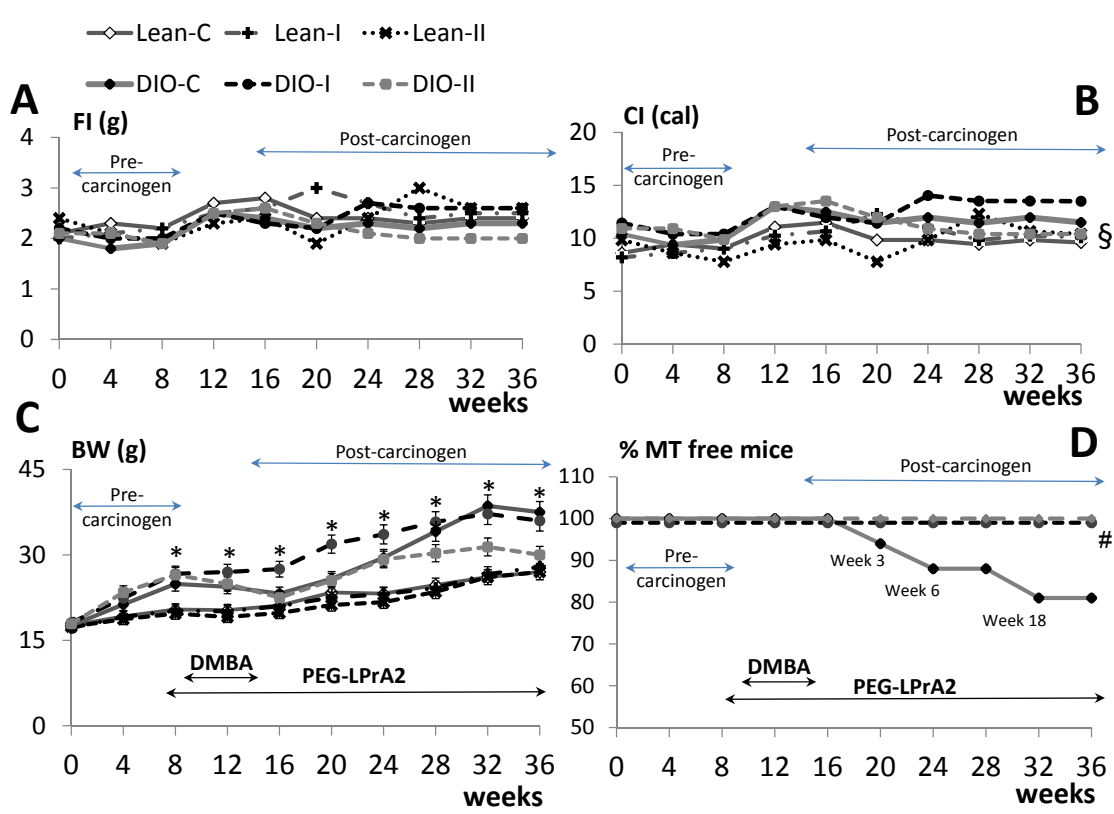

Figure 1: Impact of diets and PEG-LPrA2 treatment on food intake ( $\mathrm{FI}$, grams), caloric intake (Cl, calories), body weight (BW, grams) and incidence of mammary tumors (MT) in C57BL/6J mice challenged with DMBA. (A) Changes in weekly FI, (B) Cl and (C) \% BW change respect to BW of lean control (L-C) along the experimental period. Lean ( $n=20$; fed low-fat diet: $10 \%$ cal-fat) and DIO-mice ( $n=28$; fed high-fat diet, HFD: $55 \%$ cal-fat) were treated with PEG-LPrA2 [once (I) or twice (II) a week] or received saline injections. (D) Cumulative percentage of MT-free mice. The Kaplan-Meier curves represent DIO-mice treated with saline or PEG-LPrA2 (DIO-I and DIO-II). PEG-LPrA treatment started 2 weeks previously to DMBA challenge (6-weeks $1 \mathrm{mg} /$ week) and continued to the end of the experiment (32 weeks). MTs were detected in DIO-C mice at weeks 3,6 and 18. Obesity was defined as: BW $\mathrm{DIO}_{\mathrm{DIO}}>\mathrm{BW}_{\mathrm{L}-\mathrm{C}}+2 \mathrm{SD}$. (§) $\mathrm{p}<0.01$ when comparing Cl in DIO-C and DIO-I to DIO-II, L-C, L-I and L-II. $\left(^{*}\right) p<0.05$ when comparing BW of lean to DIO-mice. (\&) $p<0.05$ when comparing BW of DIO-C and DIO-I to DIO-II mice. (\#) $p=0.03$ (likelihood-ratio Chi2 test) when comparing MT incidence in non-treated (DIO-C) to PEG-LPrA treated mice (DIO-I and DIO-II).

treated, lean untreated, DIO treated, and DIO untreated; Chi2 $\mathrm{p}=0.04)$; (2) comparing MT-free time for lean and DIO-mice irrespective of PEG-LPrA2 treatment (Chi2 p=0.13) and (3) comparing MT-free time for DIO-mice treated or untreated with PEG-LPrA2 irrespective of mice weight (Chi2 $\mathrm{p}=0.03)$.

As expected, lean mice fed low-fat diet treated or untreated with PEG-LPrA2 did not develop MT. Cumulative percentage of MTfree DIO-mice using a Kaplan-Meier curve is shown in figure 1D. Interestingly, the development of MT in C57BL/6J female mice (that are DMBA non-responsive without hormonal stimulation) $[18,29]$ was dependent on HFD intake and obesity. MT development was only detected in C57BL/6J mice (incidence 21\%;3/14) in the obese control group (PEG-LPrA2 untreated DIO-C mice). Moreover, the onset of MT in DIO-C mice was accelerated when compared to previously reported MT onset in hormonally stimulated C57BL/6J mice (after 36 weeks of DMBA challenge) [29]. Latencies of MT in DIO-C mice were 3, 6 and 18 weeks after the DMBA last dose (Figure 1D). Remarkably, PEG-LPrA2 treatment initiated two weeks before DMBA challenge and applied either once or twice a week completely prevented the development of MT in DIO-mice ( $\mathrm{p}=0.03$; Figure $1 \mathrm{D}$ and Table 2 ).

\section{MT and other tumors induced by DMBA}

Only one MT per mouse was found in each case. Pathological analysis showed that all DMBA-MT were adenocarcinomas with squamous epithelial differentiation. Lymphomas and skin tumors (papilloma and neoplasia) were also found in several mouse subgroups irrespectively of diets fed or PEG-LPrA2 treatments (Table 2). However, L-C and DIO-I did not develop lymphoid tumors. These data suggest that HFD intake, obesity or leptin signaling were not significantly linked to DMBA-induced non-MTs in this mouse model.

\section{DMBA-induced MTs overexpressed several leptin-targeted} molecules

DMBA-MTs and mammary glands from DIO-mice untreated (DIO-C) and treated with PEG-LPrA2 were analyzed by IHC. Notably, several pro-angiogenic, inflammatory, anti-apoptotic molecules and AhR within were overexpressed in MT when compared to mammary glands from DIO-mice treated with PEG-LPrA2 (DIO-I or DIO-II) (Figure 2). AhR (Figure 2B), leptin (Figure 2F), Notch1 (Figure 2L), Notch4 (Figure 2R) and Bcl-2 (Figure 2Y) were strongly expressed in MT. In addition, OB-R (Figure 2D), VEGFR2 (Figure 2H), IL-1R tI (Figure 2I), Notch3 (Figure 2P) and JAG1 (Figure 2T) were moderately expressed in MT. In contrast, Notch2 (Figure 2M) and Survivin (Figure $2 \mathrm{~W}$ ) were weakly expressed in MT.

\begin{tabular}{|c|c|c|c|c|}
\hline \multirow{2}{*}{ Mouse group } & & & BW & \\
\cline { 3 - 5 } & $\mathrm{n}$ & Initial & Final & Carcass \\
\hline L-C & 6 & $17.9 \pm 0.6$ & $25.0 \pm 4.1$ & $17.8 \pm 1.9$ \\
\hline L-I & 7 & $17.2 \pm 1.4$ & $24.7 \pm 4.9$ & $17.2 \pm 3.6$ \\
\hline L-II & 7 & $17.4 \pm 0.8$ & $24.4 \pm 3.9$ & $16.2 \pm 3.2$ \\
\hline DIO-C & 11 & $17.8 \pm 1.1$ & $35.5 \pm 8.4^{\mathrm{f}}$ & $23.3 \pm 4.8$ \\
\hline DIO-I & 7 & $18.1 \pm 0.8$ & $34.9 \pm 9.7^{\mathrm{g}}$ & $19.4 \pm 3.4$ \\
\hline DIO-II & 7 & $17.8 \pm 0.6$ & $25.6 \pm 4.9^{\mathrm{b}}$ & $17.8 \pm 3.2^{\mathrm{d}}$ \\
\hline DIO-MT & 3 & $17.4 \pm 1.2$ & $22.1 \pm 3.7^{\mathrm{a}, \mathrm{c}}$ & $15.4 \pm 1.6^{\mathrm{e}}$ \\
\hline
\end{tabular}

Term definitions:

BW: body weight (grams, g).

DIO-MT: DIO-mice hosting mammary tumors.

"a": Net BW of DIO-MT (BW-DIO mice subtracting mammary tumor weights). Statistical significance: $b=p \leq 0.01$ (DIO-C vs DIO-II); $c=p \leq 0.01$ (DIO-C vs DIOMT); $d=p \leq 0.02$ (DIO-C vs DIO-II); $e=p \leq 0.02$ (DIO-C vs DIO-MT); $f=p \leq 0.01$ (L-C vs DIO-C); $g=p \leq 0.04$ (L-C vs DIO-I).

Table 1: Effects of leptin antagonist treatment on body weight of DMBA-mice. 

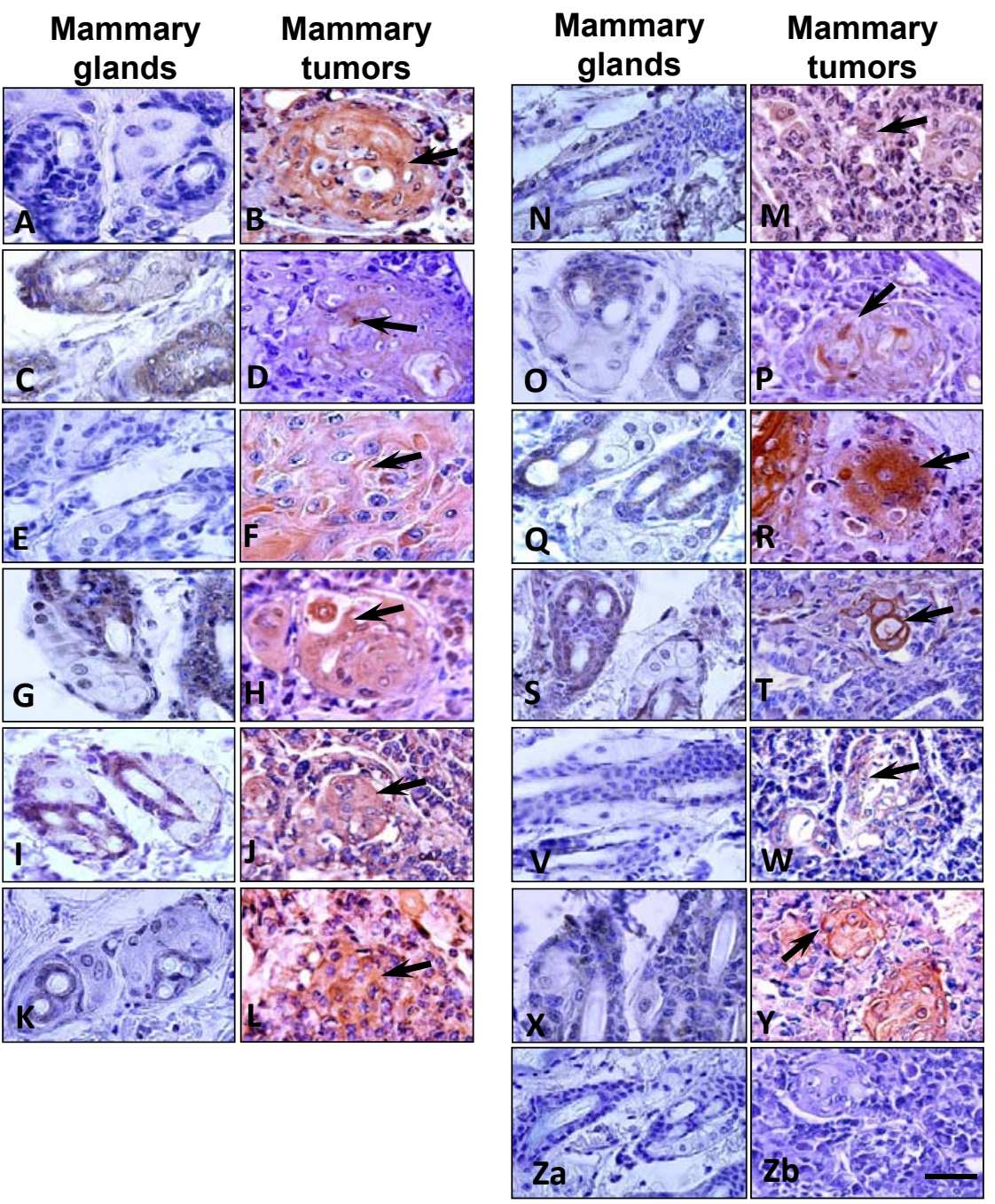

Figure 2: Representative pictures from the immunohistochemical determination of several molecules expressed by mammary glands from mice treated with $P E G$ LPrA2 and mammary tumors (DMBA-MT) developed in DIO-C (untreated mice), respectively. (A, B) AhR; (C, D) OB-R; (E, F) Leptin; (G, H) VEGFR-2; (I, J) IL-1R tl; (K, L) Notch1; (M, N) Notch2; (O, P) Notch3; (Q, R) Notch4; (S, T) JAG1; (V, W) Survivin; (X, Y) Bcl-2; (Za, Zb) Negative control.

Similar results were obtained from mammary glands without MT from the same animal (not included). Arrows indicate positive staining in MTs. Bar $=200 \mu \mathrm{m}$.

WB analysis further revealed that MT overexpressed several molecules with putative roles in breast cancer (i.e., Notch family: receptors Notch1-4; ligands: JAG-1, DLL-4 and targeted-molecules: Survivin and Hey2) when compared to mammary glands from untreated mice or treated with PEG-LPrA2 (Figure 3A). Additionally, MT showed higher levels of AhR, pro-angiogenic and inflammatory (HIF-1 $\alpha$, NFkB p105, VEGF/VEGFR-2 and IL-1R tI) and anti-apoptotic molecules (Bcl-2) when compared to mammary glands from untreated DIO-mice or treated with PEG-LPrA2 (Figure 3A). Remarkably, PEGLPrA2 treatment to DIO-mice reduced the mammary gland levels of numerous molecules (i.e., AhR, OB-R, IL-1R tI, HIF-1a, JAG1 and NotchNICD1) when compared to mammary glands from untreated DIO-C mice without MT (Figure 3B).

\section{Levels of cytokines and metabolic syndrome markers}

In general, lean mice treated or untreated with PEG-LPrA2 showed no changes in the plasmatic or mammary tissue levels of leptin, VEGF, Adiponectin and IL-1 (Figures 4A and 4B). However, lean mice treated twice a week with PEG-LPrA2 (L-II) had lower levels of IL-
$1 \alpha$ in mammary tissues (Figure 4B). Similarly, DIO-mice without MT (untreated or treated with PEG-LPrA2) showed no differences in the plasmatic or mammary tissue levels of leptin, VEGF, Adiponectin and IL-1 (Figures 4C and 4D). However, DIO-mice hosting MT had significantly higher levels of VEGF in plasma and tumors tissues (Figures 4C and 4D). In addition, DIO-mice with MT had lower plasmatic levels of leptin (Figure 4C), which corresponded to their significant loss of BW (Table 1). These mice had higher levels of leptin and IL-1 $\alpha$ in MT tissue (Figure 4D). Analysis of glucose, insulin and estradiol levels did not reveal differences between the groups of obese or lean mice (Table 3). However, DIO-II mice showed a trend of lower levels of glucose and insulin when compared to DIO-control (Table 3).

\section{Discussion}

According to the WHO, more than $30 \%$ of cancer deaths can be prevented by modifying or avoiding key risk factors [30]. Among these factors overweight/obesity, physical inactivity and consuming Western diets could play important roles for both cancer development and prognosis, increasing the risk for overall and breast cancer mortality 

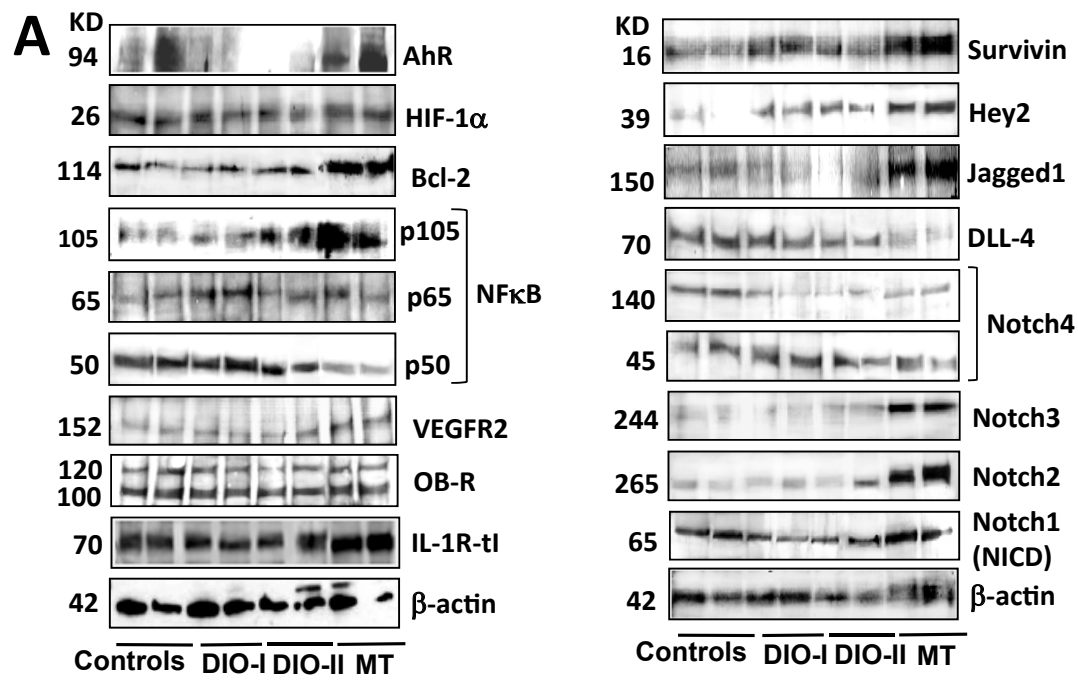

\section{B Protein/ $\beta$-actin ratio $(\%)$}

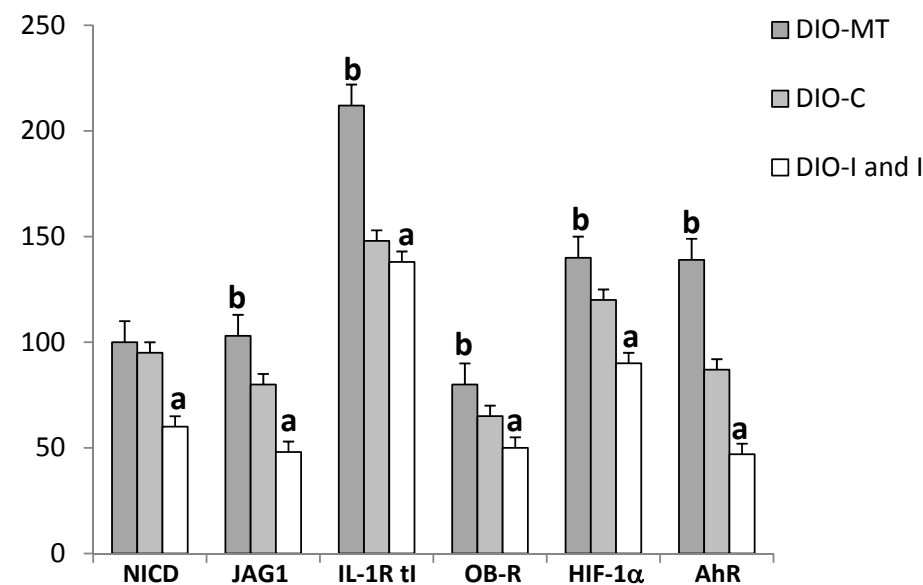

Figure 3: Expression levels of several leptin-targeted molecules in DMBA-induced MT and mammary tissues from controls and PEG-LPrA2 treated mice. (A) Western blot (WB) analysis of leptin-related molecules, (B) quantitative analysis of protein levels for Notch (receptors, ligands and target genes) in malignant and tumor-free mammary tissues. Quantitative WB data were calculated from densitometric analysis of bands with the NIH image program. The values were normalized to $\beta$-actin as a control. (a) $p<0.05$ when comparing levels of protein in mammary glands from DIO-mice untreated (DIO-C) to DIO-mice treated with PEG-LPrA. (b) $p<0.05$ when comparing levels of protein in MT to those in mammary glands from DIO-mice untreated (DIO-C) or treated with PEG-LPrA. Data (mean \pm standard error) representative results derived from a minimum of 3 independent experiments.

by approximately $30 \%$ [31]. Obesity negatively impacts the survival of breast cancer patients regardless of menopausal status, as it has been positively associated with increased risk of recurrence and increased proportion of breast cancer unresponsive to estrogens [9].

Higher incidence and shorter onset of DMBA-induced mammary tumors are mainly found in rats compared to mice [14]. Moreover, in contrast to mice, carcinogen-induced MTs can be developed in rats without additional hormonal stimulation $[14,15]$. These data clearly suggest that DMBA-MT development is species-dependent.

Several mouse strains under hormonal stimulations have been used to study the effects of various compounds and diets on MT development $[13,16-18,26,29,32-35]$. However, scarce information on the effects and potential mechanisms involved in the relationships between HFD and carcinogen-induced MT in mice is available [16].

We purposely chose the C57BL/6J mice, which are non-responsive to DMBA-induced MT in absence of hormonal stimulation [18]. C57BL/6J mice are particularly resistant to developing DMBA-MT, even with the use of pituitary isografts (MT: 27-38\% after 9 months) [29]. However, these mice are useful models to study the effects of obesity on cancer growth. C57BL/6J mice have a marked ability to gain weight and develop obesity when fed HFD [36].

In the present investigation C57BL/6J lean mice fed a low-fat diet were used as control. Ninety three percent of C57BL/6J-obesity prone mice (non-hormonal stimulated) fed HFD developed obesity. Later, these mice were challenged with the most effective reported dosage of DMBA (6-mg/6 weeks) [16]. It is known that C57BL/6J mice did not develop MT after 18 months of DMBA challenge when they are not hormonal stimulated [18]. Interestingly, present data show that only DIO-mice developed MT (21\% incidence), suggesting positive, but marginal effects of HDF and obesity on the incidence of DMBA-MT. However, significantly earlier MT onset was detected 

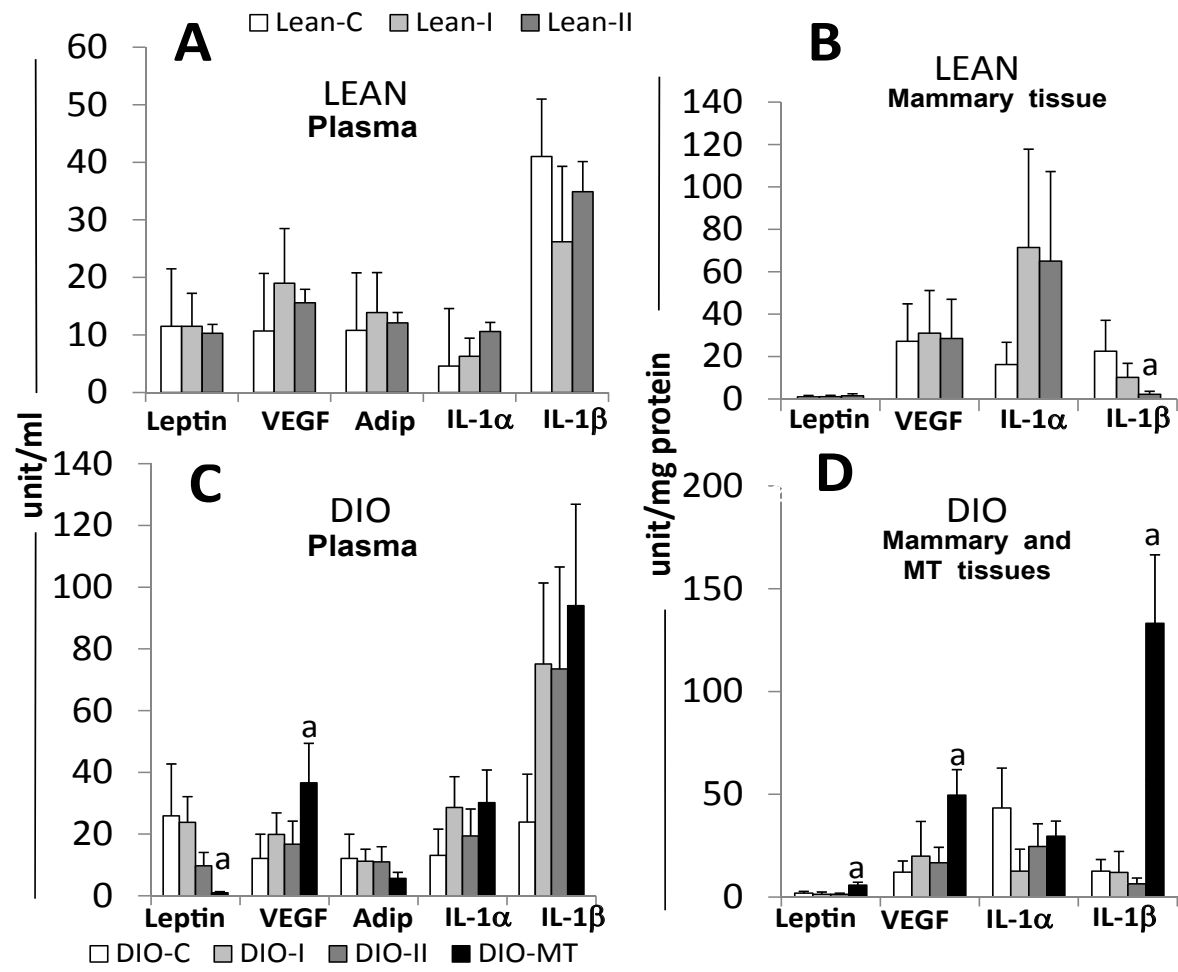

Figure 4: Plasma and tissue levels of leptin and leptin-related cytokines in mice challenged with DMBA. Lean ( $\mathrm{n}=20$; fed low-fat diet: $10 \%$ cal-fat) and DIO-mice ( $\mathrm{n}=28$ fed high-fat diet, HFD: $55 \%$ cal-fat) were treated with PEG-LPrA2 [once (I) or twice (II) a week] or received saline injections for 32 weeks. Levels of leptin (ng/ml); VEGF ( $\mathrm{ng} / \mathrm{ml})$; adiponectin $\left(\mathrm{ng} / \mathrm{ml} \times 10^{3}\right)$; and IL-1 $\alpha$ and $\beta(\mathrm{pg} / \mathrm{ml} \times 10)$ in plasma (A and C) from lean and DIO-mice, respectively, as determined by ELISA. Levels of leptin (ng/ml/mg-protein); VEGF (ng/ml/mg-protein); and IL-1 $\alpha$ and $\beta$ (pg/ml/mg-protein $\times 10)$ in mammary and MT tissue homogenates (B and D) from lean and DIO-mice, respectively, as determined by ELISA. (a) $p<0.05$ when comparing levels to lean (L-C) and obese controls (DIO-C) without MT. Data (mean \pm standard error) representative results derived from triplicate determinations.

(3-18 weeks after DMBA), which sharply contrasts to previous data reported for hormonal-stimulated C57BL/6J mice (18-36 weeks; MT mean incidence: 32\%) [29]. Present observations suggest that HFD and obesity accelerated the oncogenic transformation of the mammary glands initially elicited by DMBA. This is in agreement with previous observations showing dietetic caloric restriction increased survival (36\%) of non-hormonal stimulated C57BL/6J mice treated with DMBA [18].

Some contradictory data on HFD and DMBA-MT have been reported in rodents, however. BALB/c female mice fed HFD (20\% fat; $37 \%$ cal-fat) and treated with 6-mg DMBA dose showed $10 \%$ higher number of breast tumors than controls fed a low-fat diet (5\% fat; $9.3 \%$ cal-fat), which was unrelated to lipid peroxidation [37]. In contrast, $75 \%$ of FVB mice (fed a low-fat diet by 8.5 months of age) had breast tumors, which suggested genetic background could play a role in DMBA-induced MT [5]. High incidence of DMBA-derived breast tumors in Sprague-Dawley female rats fed a HFD was reduced by feeding iso-fat diet with $25 \%$ calorie restriction [38]. These effects were supposedly related to decreased serum insulin and IGF-I levels [15].

However, despite all efforts made to date, the effect of caloric dietary restriction on breast cancer is still poorly understood [15,18,30,3841]. Moreover, the role of specific dietary components or patterns in accelerating breast cancer outcomes is not-well understood [39]. Indeed, modification of dietary pattern in postmenopausal women designed to decrease the fat intake did not result in a statistically significant reduction in invasive breast cancer risk [40].
Accumulating evidence suggests that leptin secreted by adipose and cancer tissues increases the development of breast cancer. A positive correlation between the levels of expression of leptin and its receptor, OB-R, and bad prognosis of breast cancer has been reported [42]. Here we provide data suggesting that leptin signaling may be essential for the development of DMBA-induced MT in DIO-C57BL/6J mice fed a HDF.

We found a trend for positive correlation between DMBA-MT and intact leptin-signaling axis $(0 \% ; 0$ of 14 ; Pearson Chi2, $\mathrm{p}=0.06$; Likehood-ratio Chi2, $\mathrm{p}=0.03$ ). This was only detected in DIO-mice showing higher levels of leptin and treated with the leptin antagonist. No previous reported data on leptin actions is available from DMBAMT mouse models. However, high levels of leptin were linked to azoxymethane (AOM)-induced colon carcinogenesis in mice [43]. In contrast, obese Zucker rats (that exhibit leptin resistance due to decreased functional OB-R) [44] were more susceptible to DMBAinduced tumorigenesis than lean controls [45]. In addition, adiposity and leptin levels were not associated in a carcinogenic-induced coloncancer Sprague-Dawley rat model [46]. These disparate results on the role of obesity, leptin and dietary fat could be due to the use of different rodent strains and carcinogenics targeting specific organs.

The reduction of BW/adiposity and leptin levels were earlier suggested to play a role in DMBA-induced [38] and in spontaneous MT developed by MMTV-TGF- $\alpha$ mice [28], respectively. However, to the best of our knowledge this is the first report showing direct evidence on total prevention of DMBA-MT by specifically targeting leptin signaling. 
Citation: Gillespie C, Quarshie A, Penichet M, Gonzalez-Perez RR (2012) Potential Role of Leptin Signaling in DMBA-induced Mammary Tumors by Non-Responsive C57BL/6J Mice Fed a High-Fat Diet. J Carcinogene Mutagene 3:132. doi:10.4172/2157-2518.1000132

\begin{tabular}{|c|c|c|c|c|c|}
\hline & \multicolumn{2}{|c|}{ LOW-FAT DIET (10\% Kcal-fat) } & \multicolumn{2}{c|}{ HIGH-FAT DIET (DIO; 55\% Kcal-fat) } \\
\hline & Untreated & PEG-LPrA2 treated & Untreated & DEG-LPrA2 treated \\
\hline & Lean-C & Lean-I & Lean-II & DIO-C & DIO-II \\
\hline Mammary tumors & $0 / 6(0 \%)$ & $0 / 7(0 \%)$ & $0 / 7(0 \%)$ & $3 / 14(21 \%)$ & $0 / 7(0 \%)$ \\
\hline Lymphoid tumors & $0 / 6(0 \%)$ & $1 / 7(14 \%)$ & $2 / 7(29 \%)$ & $3 / 14(21 \%)$ & $0 / 7(0 \%)$ \\
\hline Skin tumors & $4 / 6(67 \%)$ & $4 / 7(57 \%)$ & $1 / 7(14 \%)$ & $8 / 14(57 \%)$ & $3 / 7(43 \%)$ \\
\hline
\end{tabular}

Table 2: Incidence of mammary and non-mammary tumors in mice challenged with DMBA, treated and untreated with PEG-LPrA2, and fed with control or DIO-diets.

\begin{tabular}{|c|c|c|c|c|c|c|c|}
\hline \multirow{2}{*}{$\begin{array}{l}\text { Mouse } \\
\text { group }\end{array}$} & \multirow[b]{2}{*}{$n$} & \multicolumn{2}{|c|}{ Glucose } & \multicolumn{2}{|c|}{ Insulin } & \multicolumn{2}{|c|}{ E2 } \\
\hline & & Raw & BW-N & Raw & BW-N & Raw & BW-N \\
\hline L-C & 6 & $173.7 \pm 28.8$ & $6.7 \pm 1.7$ & $78.2 \pm 22.6$ & $3.0 \pm 1.1$ & $2.4 \pm 1.1$ & $0.1 \pm 0.0$ \\
\hline L-I & 7 & $186.7 \pm 21.7$ & $7.1 \pm 1.3$ & $95.6 \pm 15.4$ & $4.3 \pm 1.5$ & $7.5 \pm 7.8$ & $0.3 \pm 0.3$ \\
\hline L-II & 7 & $162.5 \pm 19.8$ & $6.5 \pm 0.9$ & $96.1 \pm 39.0$ & $4.1 \pm 1.6$ & $4.1 \pm 4.0$ & $0.2 \pm 0.2$ \\
\hline DIO-C & 11 & $228.8 \pm 89.5$ & $6.4 \pm 2.8$ & $85.1 \pm 29.3$ & $2.8 \pm 1.1$ & $6.2 \pm 8.8$ & $0.2 \pm 0.0$ \\
\hline DIO-I & 7 & $216.9 \pm 58.6$ & $7.0 \pm 3.2$ & $120.6 \pm 48.1$ & $4.0 \pm 1.9$ & $4.1 \pm 2.1$ & $0.1 \pm 0.0$ \\
\hline DIO-II & 7 & $174.9 \pm 51.3$ & $6.4 \pm 1.5$ & $70.6 \pm 13.2$ & $2.6 \pm 0.4$ & $4.3 \pm 3.1$ & $0.2 \pm 0.1$ \\
\hline DIO-MT & 3 & $333.9 \pm 252.9$ & $14.3 \pm 11.7$ & $70.6 \pm 0.0$ & $2.9 \pm 0.2$ & $4.7 \pm 3.2$ & $0.2 \pm 0.2$ \\
\hline
\end{tabular}

Term definitions:

DIO-MT: DIO-mice hosting mammary tumors.

Raw: levels of plasmatic parameters not adjusted to body weight [(glucose, $\mathrm{mg} / \mathrm{dL})$; (insulin, pmol/L) and (E2, estradiol, $\mathrm{pg} / \mathrm{mL})]$.

BW-N: levels of plasmatic parameters normalized to body weight in grams [(glucose, $\mathrm{mg} / \mathrm{dL} / \mathrm{g}$ ), (insulin, $\mathrm{pmol} / \mathrm{L} / \mathrm{g}) \mathrm{and}(\mathrm{estradiol}, \mathrm{pg} / \mathrm{mL} / \mathrm{g}$ ).

Table 3: Effects of leptin antagonist treatment on plasmatic levels of glucose, insulin and estradiol in DMBA-mice.

Notch signaling activation, a hallmark of breast cancer development [47] is also required for DMBA-induced MT [48]. Mammary glands from DIO-mice treated with PEG-LPrA2 showed lower levels of several leptin-related molecules (NotchNICD1, JAG1, IL-1R tI; OB-R, HIF$1 \alpha$ and $\mathrm{AhR}$ ) [49] than mammary glands from untreated mice. These results suggest that Notch signaling could be involved in DMBA-MT. Moreover, these data reinforce the idea that an intact leptin-signaling axis is needed for the increase of JAG1 (a Notch ligand) [47] in DMBAinduced MTs.

In addition, DMBA-induced pre-invasive MT requires angiogenesis via VEGF/VEGFR-2 mechanism [48]. Indeed, the inhibition of VEGFR-2 signaling abrogates the development of DMBA-MT in rats [48]. In line with these data, leptin induced a complex crosstalk in breast cancer, NILCO (Notch-IL-1-leptin crosstalk outcome), that upregulates VEGF/VEGFR-2 [49]. Moreover, leptin can activate VEGFR-2 in absence of VEGF [49]. Therefore, current data suggest that the development of DMBA-MT in C57BL/6J obese mice probably involves leptin-induced activation of NILCO and upregulation of VEGF/VEGFR-2 [49].

Overall, present data suggest obesity and HFD accelerate DMBAinduced MT, and that leptin signaling may be essential for malignant transformation of mammary glands of $\mathrm{C} 57 \mathrm{BL} / 6 \mathrm{~J}$ mice. Therefore, inhibition of leptin signaling may provide novel prevention strategy for carcinogenic-induced breast cancer, particularly, in overweight/obese individuals feeding high-fat diets.

\section{Limitation of the Study}

Although, we acknowledge the small sample size of the study, however, the data is compelling enough for hypothesis generation to support a bigger study to validate the findings of the present investigation.

\section{Disclosure of Potential Conflicts of Interest}

The authors declare that they have no conflicts of interest. RRGP is an inventor of the Boston Biomedical Research Institute's patent Leptin Peptide Antagonists (US Patent 7407929, Application No. 10/841,218; International application No. PCT/US 05/15198). No financial benefits have been derived from this patent.

\section{Grant Support}

This work was supported by $\mathrm{NIH} / \mathrm{NCl} 5 \mathrm{SC} 1 \mathrm{CA} 138658-03$ and the Georgia
Cancer Coalition Distinguished Cancer Scholar Award (to RRGP); and NIH/2G12RR003034-26; U54 MSM/TU/UAB Cancer Center Partnership. This research was supported, in part, facilities and support services at Morehouse School of Medicine.

\section{References}

1. Rundle A, Tang D, Hibshoosh H, Estabrook A, Schnabel F, et al. (2000) The relationship between genetic damage from polycyclic aromatic hydrocarbons in breast tissue and breast cancer. Carcinogenesis 21: 1281-1289.

2. Denison MS, Nagy SR (2003) Activation of the aryl hydrocarbon receptor by structurally diverse exogenous and endogenous chemicals. Annu Rev Pharmacol Toxicol 43: 309-334.

3. Kimura K, Satoh K, Kanno A, Hamada S, Hirota M, et al. (2007) Activation of Notch signaling in tumorigenesis of experimental pancreatic cancer induced by dimethylbenzanthracene in mice. Cancer Sci 98: 155-162.

4. Abdelrahim M, Smith R, 3rd, Safe S (2003) Aryl hydrocarbon receptor gene silencing with small inhibitory RNA differentially modulates Ah-responsiveness in MCF-7 and HepG2 cancer cells. Mol Pharmacol 63: 1373-1381.

5. Currier N, Solomon SE, Demicco EG, Chang DL, Farago M, et al. (2005) Oncogenic signaling pathways activated in DMBA-induced mouse mammary tumors. Toxicol Pathol 33: 726-737.

6. Dusing-Swartz S, Medina D, Butel JS, Socher SH (1979) Mouse mammary tumor virus genome expression in chemical carcinogen-induced mammary tumors in low- and high-tumor-incidence mouse strains. Proc Natl Acad Sci U S A 76: $5360-5364$

7. www.epa.gov/osw/hazard (2012)

8. Falck F J, Ricci A, Jr., Wolff MS, Godbold J, Deckers P. (1992) Pesticides and polychlorinated biphenyl residues in human breast lipids and their relation to breast cancer. Arch Environ Health 47: 143-146.

9. Daling JR, Malone KE, Doody DR, Johnson LG, Gralow JR, et al. (2001) Relation of body mass index to tumor markers and survival among young women with invasive ductal breast carcinoma. Cancer 92: 720-729.

10. Whiteman MK, Hillis SD, Curtis KM, McDonald JA, Wingo PA, et al. (2005) Body mass and mortality after breast cancer diagnosis. Cancer Epidemio Biomarkers Prev 14: 2009-2014.

11. Moon RC (1969) Relationship between previous reproductive history and chemically induced mammary cancer in rats. Int J Cancer 4: 312-317.

12. Russo J, Russo IH (1980) Susceptibility of the mammary gland to carcinogenesis. II. Pregnancy interruption as a risk factor in tumor incidence. Am J Pathol 100: 497-512. 
Citation: Gillespie C, Quarshie A, Penichet M, Gonzalez-Perez RR (2012) Potential Role of Leptin Signaling in DMBA-induced Mammary Tumors by Non-Responsive C57BL/6J Mice Fed a High-Fat Diet. J Carcinogene Mutagene 3:132. doi:10.4172/2157-2518.1000132

13. Medina D, Smith GH (1999) Chemical carcinogen-induced tumorigenesis in parous, involuted mouse mammary glands. J Natl Cancer Inst 91: 967-969.

14. Klurfeld DM, Welch CB, Lloyd LM, Kritchevsky D (1989) Inhibition of DMBAinduced mammary tumorigenesis by caloric restriction in rats fed high-fat diets. Int J Cancer 43: 922-925

15. Ruggeri BA, Klurfeld DM, Kritchevsky D, Furlanetto RW (1989) Caloric restriction and 7,12-dimethylbenz(a)anthracene-induced mammary tumo growth in rats: alterations in circulating insulin, insulin-like growth factors I and II, and epidermal growth factor. Cancer Res 49: 4130-4134.

16. Lane HW, Butel JS, Howard C, Shepherd F, Halligan R, et al. (1985) The role of high levels of dietary fat in 7,12-dimethyl-benzanthracene-induced mouse mammary tumorigenesis: lack of an effect on lipid peroxidation. Carcinogenesis 6: 403-407.

17. Medina D (1974a) Mammary tumorigenesis in chemical carcinogen-treated mice. II. Dependence on hormone stimulation for tumorigenesis2. J Natl Cancer Inst 53: 223-226.

18. Lipman RD (2002) Effect of calorie restriction on mortality kinetics in inbred strains of mice following 7,12-dimethylbenz[a]anthracene treatment. J Gerontol A Biol Sci Med Sci 57: 153-157.

19. Gautron L, Elmquist JK (2011) Sixteen years and counting: an update on leptin in energy balance. J Clin Invest 121: 2087-2093.

20. Gonzalez RR, Cherfils S, Escobar M, Yoo JH, Carino C, et al. (2006) Leptin signaling promotes the growth of mammary tumors and increases the expression of vascular endothelial growth factor (VEGF) and its receptor type two (VEGF-R2). J Biol Chem 281: 26320-26328.

21. Gonzalez-Perez RR, Xu Y, Guo S, Watters A, Zhou W, et al. (2010) Leptin upregulates VEGF in breast cancer via canonic and non-canonical signalling pathways and NFkappaB/HIF-1alpha activation. Cell Signal 22: 1350-1362.

22. Rene Gonzalez R, Watters A, Xu Y, Singh UP, Mann DR, et al. (2009) Leptinsignaling inhibition results in efficient anti-tumor activity in estrogen receptor positive or negative breast cancer. Breast Cancer Res 11: 36

23. Zhou W, Guo S, Gonzalez-Perez RR (2011) Leptin pro-angiogenic signature in breast cancer is linked to IL-1 signalling. Br J Cancer 104: 128-137.

24. Gonzalez RR, Leavis PC (2003) A peptide derived from the human leptin molecule is a potent inhibitor of the leptin receptor function in rabbit endometrial cells. Endocrine 21: 185-195.

25. Phytoestrogen Reduced I 22.5-5.

26. Cox D, Oakes D (1984) Analysis of Survival Data. London: Chapman and Hall.

27. Dogan S, Hu X, Zhang Y, Maihle NJ, Grande JP, et al. (2007) Effects of highfat diet and/or body weight on mammary tumor leptin and apoptosis signaling pathways in MMTV-TGF-alpha mice. Breast Cancer Res 9: 91.

28. Medina D (1974b) Mammary tumorigenesis in chemical carcinogen-treated mice. I. Incidence in BALB-c and C57BL mice. J Natl Cancer Inst 53: 213-221.

29. Bode AM, Dong Z (2009) Cancer prevention research - then and now. Nat Rev Cancer 9: 508-516.

30. Calle EE, Rodriguez C, Walker-Thurmond K, Thun MJ (2003) Overweight, obesity, and mortality from cancer in a prospectively studied cohort of U.S. adults. N Engl J Med 348: 1625-1638.

31. Crowley MR, Frost A, Chen DT, Baffi MO, Nicola T, et al. (2006) Transforming growth factor-beta signaling helps specify tumor type in DMBA and hormoneinduced mammary cancers. Differentiation 74: 40-52.

32. Jerry DJ, Butel JS, Donehower LA, Paulson EJ, Cochran C, et al. (1994) Infrequent p53 mutations in 7,12-dimethylbenz[a]anthracene-induced mammary tumors in BALB/c and p53 hemizygous mice. Mol Carcinog 9: 175183.

33. Wolff GL, Kodell RL, Cameron AM, Medina D (1982) Accelerated appearance of chemically induced mammary carcinomas in obese yellow (Avy/A) (BALB/c X VY) F1 hybrid mice. J Toxicol Environ Health 10: 131-142.

34. Gu JW, Young E, Patterson SG, Makey KL, Wells J, et al. (2011) Postmenopausal obesity promotes tumor angiogenesis and breast cancer progression in mice. Cancer Biol Ther 11: 910-917.

35. Lane HW, Butel JS, Howard C, Shepherd F, Halligan R, et al. (1985) The role of high levels of dietary fat in 7,12-dimethylbenzanthracene-induced mouse mammary tumorigenesis: lack of an effect on lipid peroxidation. Carcinogenesis 6: 403-407.

36. Hauner H, Hauner D (2010) The Impact of Nutrition on the Development and Prognosis of Breast Cancer. Breast Care (Basel) 5: 377-381.

37. Prentice RL, Caan B, Chlebowski RT, Patterson R, Kuller LH, et al. (2006) Low-fat dietary pattern and risk of invasive breast cancer: the Women's Health Initiative Randomized Controlled Dietary Modification Trial. JAMA 295: 629642.

38. Rogozina OP, Bonorden MJ, Seppanen CN, Grande JP, Cleary MP (2011) Effect of chronic and intermittent calorie restriction on serum adiponectin and leptin and mammary tumorigenesis. Cancer Prev Res (Phila) 4: 568-581.

39. Ishikawa M, Kitayama J, Nagawa H (2004) Enhanced expression of leptin and leptin receptor (OB-R) in human breast cancer. Clin Cancer Res 10: 43254331.

40. Miyamoto S, Yasui Y, Tanaka T, Ohigashi H, Murakami A (2008) Suppressive effects of nobiletin on hyperleptinemia and colitis-related colon carcinogenesis in male ICR mice. Carcinogenesis 29: 1057-1063.

41. Brunner L, Nick HP, Cumin F, Chiesi M, Baum HP, et al. (1997) Leptin is a physiologically important regulator of food intake. Int $\mathrm{J}$ Obes Relat Metab Disord 21: 1152-1160.

42. Hakkak R, Holley AW, Macleod SL, Simpson PM, Fuchs GJ, et al. (2005) Obesity promotes 7,12-dimethylbenz(a)anthracene-induced mammary tumor development in female zucker rats. Breast Cancer Res 7: 627-633.

43. Drew JE, Farquharson AJ, Padidar S, Duthie GG, Mercer JG, et al. (2007) Insulin, leptin, and adiponectin receptors in colon: regulation relative to differing body adiposity independent of diet and in response to dimethylhydrazine. Am J Physiol Gastrointest Liver Physiol 293: 682-691.

44. Guo S, Liu M, Gonzalez-Perez RR (2011) Role of Notch and its oncogenic signaling crosstalk in breast cancer. Biochim Biophys Acta 1815: 197-213.

45. Ayyanan A, Civenni G, Ciarloni L, Morel C, Mueller N, et al. (2006) Increased Wnt signaling triggers oncogenic conversion of human breast epithelial cells by a Notch-dependent mechanism. Proc Natl Acad Sci U S A 103: 3799-3804.

46. Guo S, Gonzalez-Perez RR (2011) Notch, IL-1 and leptin crosstalk outcome (NILCO) is critical for leptin-induced proliferation, migration and VEGF/ VEGFR-2 expression in breast cancer. PLoS One 6: 21467.

47. Kovacheva VP, Davison JM, Mellott TJ, Rogers AE, Yang S, et al. (2009) Raising gestational choline intake alters gene expression in DMBA-evoked mammary tumors and prolongs survival. FASEB J 23: 1054-1063.

48. Heffelfinger SC, Yan M, Gear RB, Schneider J, LaDow K, et al. (2004) Inhibition of VEGFR2 prevents DMBA-induced mammary tumor formation. Lab Invest 84: 989-998.

49. Garonna E, Botham KM, Birdsey GM, Randi AM, Gonzalez-Perez RR, et al. (2011) Vascular endothelial growth factor receptor-2 couples cyclooxygenase-2 with pro-angiogenic actions of leptin on human endothelial cells. Plos one 6: 18823. 